\title{
sv-callers: a highly portable parallel workflow for structural variant detection in whole-genome sequence data
}

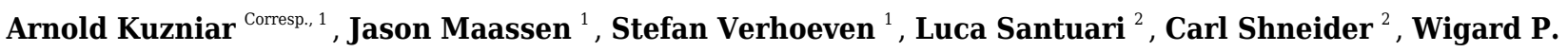 \\ Kloosterman ${ }^{2}$, Jeroen de Ridder ${ }^{\text {Corresp. } 2}$ \\ 1 Netherlands eScience Center, Amsterdam, Netherlands \\ 2 Center for Molecular Medicine, University Medical Center Utrecht, Utrecht, Netherlands \\ Corresponding Authors: Arnold Kuzniar, Jeroen de Ridder \\ Email address: a.kuzniar@esciencecenter.nl, j.deridder-4@umcutrecht.nl
}

Structural variants (SVs) are an important class of genetic variation implicated in a wide array of genetic diseases including cancer. Despite the advances in whole genome sequencing, comprehensive and accurate detection of SVs in short-read data still poses some practical and computational challenges. We present sv-callers, a highly portable workflow that enables parallel execution of multiple SV detection tools as well as provide users with example analyses of detected SV callsets in a Jupyter Notebook. This workflow supports easy deployment of software dependencies, configuration and addition of new analysis tools. Moreover, porting it to different computing systems requires minimal effort. Finally, we demonstrate the utility of the workflow by performing both somatic and germline SV analyses using different high-performance computing systems. 


\section{1 sv-callers: a highly portable parallel workflow for}

2 structural variant detection in whole-genome

3 sequence data

Arnold Kuzniar ${ }^{1}$, Jason Maassen ${ }^{1}$, Stefan Verhoeven ${ }^{1}$, Luca Santuari², Carl Shneider ${ }^{2}$, Wigard P. Kloosterman ${ }^{2}$, Jeroen de Ridder ${ }^{2}$

${ }^{1}$ Netherlands eScience Center, Amsterdam, The Netherlands,

Corresponding Authors:

13 Arnold Kuzniar

14 Science Park 140, 1098 XG Amsterdam, The Netherlands

Jeroen de Ridder 


\section{Abstract}

39 Structural variants (SVs) are an important class of genetic variation implicated in a wide array of genetic diseases including cancer. Despite the advances in whole genome sequencing, comprehensive and accurate detection of SVs in short-read data still poses some practical and computational challenges. We present $s v$-callers, a highly portable workflow that enables parallel execution of multiple SV detection tools as well as provide users with example analyses of detected SV callsets in a Jupyter Notebook. This workflow supports easy deployment of software dependencies, configuration and addition of new analysis tools. Moreover, porting it to different computing systems requires minimal effort. Finally, we demonstrate the utility of the workflow by performing both somatic and germline SV analyses using different highperformance computing systems.

\section{Introduction}

Structural variants (SVs) such as deletions, insertions and duplications account for a large part of the genomic diversity among individuals and have been implicated in many diseases including cancer. With the advent of novel DNA sequencing technologies, whole genome sequencing (WGS) is becoming an integral part of cancer diagnostics that can potentially enable tailored treatments of individual patients (Stratton, 2011). Despite advances in large-scale cancer genomics projects (such as the TCGA and PCAWG of the International Cancer Genome Consortium; https://icgc.org/), systematic and comprehensive analysis of massive genomic data, in particular the detection of structural variations (SVs) in genomes, remains challenging due to computational and algorithmic limitations (Alkan, Coe \& Eichler, 2011; Yung et al., 2017; Ma et al., 2018; Gröbner et al., 2018).

61 Recent tools for somatic and germline SV detection (callers) exploit more than one type of 62 information present in WGS data (Lin et al., 2016). For example, DELLY (Rausch et al., 2012) 63 relies on split reads and discordant read pairs while LUMPY (Layer et al., 2014) additionally 64 utilizes read depth information. Furthermore, callers such as Manta (Chen et al., 2016) and GRIDSS (Cameron et al., 2017) also incorporate short-read assembly. To obtain a more comprehensive and/or accurate callset, ensemble approaches have yielded promising results (English et al., 2015; Mohiyuddin et al., 2015; Becker et al., 2018; Fang et al., 2018). In such an approach, i) a range of SV callers are executed, and ii) their results are combined into a single callset. While this approach has been demonstrated to improve SV callsets, the step (i) poses a 
71 infrastructure and/or adding new SV callers (as they become available) is far from

72 straightforward.

73 A common practice to couple multiple tools together is by monolithic "wrapper" scripts (English

74 et al., 2015; Fang et al., 2018) and, to a lesser extent, by a workflow system. The latter is a

75 recommended approach to improve the extensibility, portability and reproducibility of data-

76 intensive analyses (Leipzig, 2016). Specifically, multiple command-line tools, usually written in

77 different languages, are integrated in a workflow (instance) that is defined either by an implicit

78 syntax (Köster \& Rahmann, 2012; Holmes \& Mungall, 2017) or by an explicit syntax (Afgan et

79 al., 2018; Vivian et al., 2017). In addition, a workflow system might support parallel execution

80 of tasks through a batch scheduler that distributes the tasks across nodes of a high-performance computing (HPC) system. For example, Snakemake is a well-established generic workflow system inspired by the GNU Make build automation tool (Köster \& Rahmann, 2012). It requires

a "recipe" with implicit rules to generate output from input files. Moreover, Snakemake supports

84 Conda package manager and light-weight containers such as Docker (https://www.docker.com/)

85

86

87

88

89

90

91

92

93

94

95

96

97

98

99

100

101

102

103

104

or Singularity (Kurtzer, Sochat \& Bauer, 2017), Common Workflow Language (CWL, Amstutz et al., 2016) and parallel execution in HPC cluster (via DRMAA, http://www.drmaa.org/) or cloud environments (via Kubernetes, https://kubernetes.io/). Furthermore, a workflow developed on one system is not necessarily portable to or easily reusable on another system due to the complexity of software environments, system usage policies (e.g., lack of root privilege or absence of Docker) and/or the use of different batch schedulers. Typically, compute clusters rely on batch schedulers such as Grid Engine, Slurm or Torque to manage resources and to distribute tasks over the available nodes. As a result, workflows need to be adapted to use scheduler- or file transfer-specific commands, which makes the distribution of compute tasks across (heterogeneous) systems cumbersome.

To alleviate these problems, we developed sv-callers, a user-friendly, portable and scalable workflow based on the Snakemake and Xenon (middleware) software. The workflow includes state-of-the-art somatic and germline SV callers, which can be easily extended, and runs on HPC clusters or clouds with minimal effort. It supports all the major SV types (i.e., deletions, insertions, inversions, duplications and translocations) as detected by the individual callers. In this paper, we focus on the implementation and computational performance of the workflow as well as provide examples to improve the reproducibility of downstream analyses.

\section{Methods}

\section{Experimental data and setup}

Peer) reviewing PDF | (2019:04:37207:2:0:NEW 3 Nov 2019) 
105 The sv-callers workflow was tested with two human WGS datasets, one for the single-sample 106 mode (i.e., germline SV detection) and the other for the paired-sample mode (i.e., somatic SV

107 detection in tumor with matched normal). The benchmark data were obtained by Illumina HiSeq 1082500 sequencing of the NA12878 genome (148 GiB BAM file, downsampled to 30x coverage) 109 released by the NIST Genome in a Bottle Consortium (Zook et al., 2016). The cell lines data 110 were obtained by Illumina HiSeq X Ten sequencing of a cancer cell line derived from malignant 111 melanoma (COLO829, EFO:0002140) at 90x coverage (ENA:ERX2765496, 184 GiB BAM file)

112 and a matching control, lymphoblastoid cell line (EFO:0005292) at 30x coverage

113 (ENA:ERX2765495, 67 GiB BAM file). Illumina short reads were mapped to the human 114 reference genome (i.e., b37 and GRCh37 were used for the benchmark and the cell lines data, 115 respectively) using the BWA-MEM algorithm (Li, 2013). To increase the amount of jobs, the 116 WGS samples in BAM files were copied ten times, resulting in 140 jobs per dataset (of which 80 117 and 60 were SV calling and post-processing jobs, respectively). This allowed us to reliably

118 estimate compute resources used as well as to assess if the results are correct and/or reproducible 119 across the sample copies using the UpSet(R) plots (Conway et al., 2017).

120

\section{Structural variation detection}

122 Four SV callers were included in the workflow namely Manta (v1.1.0), DELLY (v0.7.7),

123 LUMPY (v0.2.13) and GRIDSS (v1.3.4), taking into account the state-of-the-art methods (e.g., 124 used in the ICGC projects), active development and software/documentation quality. Moreover, 125 each of the callers have been extensively validated in their respective publications. Most callers 126 make use of more than one programming language and take advantage of multi-core

127 architectures (Table 1). Further, we accommodated the Conda package manager

128 (https://conda.io/) including the 'bioconda' channel (da Veiga Leprevost et al., 2017) to ease the 129 installation of required bioinformatics software. SV detection involved the callers' default 130 settings and 'best practices' for post-processing the callsets in VCF/BCF files: i) genomic 131 regions with anomalous mappability (ENCODE:ENCFF001TDO in BED file) were filtered 132 using the SURVIVOR software (v1.0.6; Jeffares et al., 2017) with filter sub-command, ii) 133 low-quality calls and/or iii) germline calls in the paired-sample (somatic) mode were filtered 134 using BCFtools (v1.9; Li et al., 2009), and finally iv) the resulting SV calls of the four detection 135 tools were merged into one (union) set using SURVIVOR with merge sub-command with user 136 parameters defined in the workflow configuration file (analysis.yaml). In our example 137 analyses (Kuzniar \& Santuari, 2019), two SVs were considered overlapping and merged into one 138 entry if the distance between the relative breakpoints at each end is smaller than $100 \mathrm{bp}$, 
139 regardless of the SV type. Note that this threshold is within the estimated insert sizes of the 140 benchmark (552 bp) and the cell lines (518/531 bp for tumor/normal) datasets. Furthermore, the

141 germline SV calls of each tool including the derived (merged) callsets were evaluated in terms of 142 precision $[\mathrm{TP} /(\mathrm{TP}+\mathrm{FP})]$ and recall $[\mathrm{TP} /(\mathrm{TP}+\mathrm{FN})]$ using the StructuralVariantAnnotation $\mathrm{R}$ 143 package (v1.0.0; Cameron \& Dong, 2019) with high-confidence deletions published by Layer et 144 al. (2014) and Parikh et al. (2016) (i.e., the PacBio/Moleculo [ $\mathrm{n}=4095]$ and the Personalis/1000 145 Genome Project [n=2676] truth sets).

147 Workflow implementation and deployment

148 We implemented the sv-callers workflow (v1.1.0, Kuzniar et al., 2018) using the Snakemake 149 workflow system (v4.8.0; Köster \& Rahmann, 2012) and the Xenon software suite (v3.0.0). Figure 1A shows a schematic diagram of the workflow including input/output files. To perform an analysis, a user provides a CSV file with the (paired) samples and adjusts the workflow configuration file(s) accordingly. The file analysis.yaml is concerned with the analysis itself (such as the mode, reference genome, exclusion list, callers, post-processing, resource requirements, etc.) while the file environment.yaml includes software dependencies/versions. Moreover, the workflow parameters are also accessible through the command-line interface (see snakemake -C [params] argument). We developed the Xenon library (Maassen et al., 2018) and the associated command-line interface, xenon-cli (Verhoeven \& Spaaks, 2019) to provide uniform access to different batch schedulers and file transfer clients. Xenon is comparable to the Simple API for Grid Application (SAGA) (Merzky, Weidner \& Jha, 2015) and its predecessor, the Grid Application Toolkit (GAT) (Allen et al., 2005; van Nieuwpoort, Kielmann \& Bal, 2007). These APIs are client-side solutions rather than a server-side standardization attempt such as the Distributed Resource Management Application API (DRMAA) (Tröger et al., 2007). Specifically, Xenon supports several batch schedulers such as Grid Engine, Slurm and Torque, local or remote execution on a server via SSH, as well as local or remote file access via the (S)FTP and WebDAV transfer protocols, Amazon S3 cloud storage or Apache Hadoop Distributed File System (HDFS). As shown in Figure 1B, the Xenon library translates the xenon-cli commands to scheduler-specific commands used by a target cluster. When the cluster is changed or multiple clusters are used, Xenon will simply use a different translation, while the xenon-cli commands will remain essentially the same. A user needs to modify only a scheduler type or a queue name. For this reason, xenon-cli was configured as the cluster submission command for our Snakemake-based workflow, which improved the portability by making the workflow independent of the system- 
173 specific solutions (see snakemake --cluster 'xenon scheduler [type]' argument).

174 Finally, the software reliability has been ensured by regular unit and continuous integration tests

175 in the cloud (Travis CI, https://travis-ci.org/), which involve Docker images of different batch 176 systems including real test data (NA12878). After successful tests, the workflow was deployed

177 on different academic HPC systems namely the Grid Engine-based cluster at Utrecht

178 Bioinformatics Center (UBC, https://ubc.uu.nl/), and the Slurm-based Distributed ASCI

179 Supercomputer 5 (Bal et al., 2016; https://www.cs.vu.nl/das5/) and the Dutch national

180 supercomputer (Cartesius, https://userinfo.surfsara.nl/systems/cartesius/).

181

182 Results

183 The sv-callers workflow readily automated parallel execution of the tools across compute nodes 184 and enabled streamlined data analyses e.g., in a Jupyter Notebook. We tested the workflow with 185 each WGS dataset (i.e., benchmark and cell lines) on both Grid Engine- and Slurm-based HPC 186 systems, processing in total about $4 \mathrm{TiB}$ of data. Overall, the datasets were processed at different 187 rates on each system due to differences in data size and coverage, and system load or availability 188 at the time of use (data not shown). Submitted jobs of the benchmark and of the cell lines 189 datasets completed successfully (100\%) in about twelve hours and in four days, respectively.

190 Table 2 shows the compute resources used by the SV callers (on the UBC cluster). We found that 191 the detection of somatic SVs required significantly more compute time or resources than that of 192 germline SVs for the datasets used (335 versus 41 CPU core hours). In particular, the analyses by 193 DELLY or GRIDSS were computationally more costly per sample than those by Manta or 194 LUMPY (see Supplemental Data S1).

195 Further, the resulting VCF files with SV calls were checked for completeness and for overlap 196 among the callers and sample copies. Most SVs were caller-specific while the second largest 197 category of SVs were common to three of the four callers (Figure 2). Importantly, merging SV 198 calls supported by the majority of tools resulted in improved precision (but not recall) compared 199 to the best caller according to the PacBio/Moleculo (94.8\% versus 81.6\% [LUMPY]) and the 200 Personalis/1kGP (88.1\% versus 76.8\% [GRIDSS]) truth sets (Figure 3, Supplemental Data S2). 201

\section{Conclusions}

203 Relying on a single SV detection algorithm or caller is insufficient for comprehensive and 204 accurate detection of SVs in cancer genomes (English et al., 2015; Fang et al., 2018; Kosugi et 205 al., 2019). Therefore, there is an increasing need for flexible and portable bioinformatics 
206 workflows that - with minimal effort - integrate multiple SV detection and post-processing tools

207 while making efficient use of different HPC and/or cloud infrastructures. To address these needs, 208 we developed the $s v$-callers workflow according to best practices in research software (Jiménez 209 et al., 2017; Maassen et al., 2017; https://guide.esciencecenter.nl/). Further improvements to the 210 workflow itself and/or its 'backend' are possible. For example, adding multiple read mapping 211 tools to the workflow would enable analyses directly from raw sequencing data. Moreover, the

212 SV reporting step could be enhanced with interactive visualization to facilitate manual inspection 213 of SVs in genomic regions of interest. Furthermore, the SV callers' binaries (currently 214 distributed via bioconda) are suboptimal regarding the performance for target systems used, and 215 therefore the codes might benefit from further optimization (e.g., using the Intel $\mathrm{C} / \mathrm{C}++$ 216 Compiler). We aim to extend Xenon's cloud support with schedulers such as Microsoft Azure 217 Batch and Amazon AWS Batch as well as to improve the coupling between Xenon and 218 Snakemake software. Finally, the sv-callers workflow is an open-source software that is freely 219 available from the ELIXIR's bio.tools registry (https://bio.tools/sv-callers) and our Research 220 Software Directory (https://research-software.nl/software/sv-callers).

221

222

\section{Acknowledgements}

224 The authors would like to thank HPC system administrators and SURFsara consultants in 225 particular Jeroen Engelberts and Lyke Voort for technical support, and the reviewers for their 226 valuable suggestions.

227

\section{References}

229 Afgan E, Baker D, Batut B, van den Beek M, Bouvier D, Cech M, Chilton J, Clements D, Coraor 230 N, Grüning BA, Guerler A, Hillman-Jackson J, Hiltemann S, Jalili V, Rasche H, Soranzo N, 231 Goecks J, Taylor J, Nekrutenko A, Blankenberg D. 2018. The Galaxy platform for accessible, 232 reproducible and collaborative biomedical analyses: 2018 update. Nucleic Acids Research 233 46:W537-W544. DOI: 10.1093/nar/gky379.

234

Alkan C, Coe BP, Eichler EE. 2011. Genome structural variation discovery and genotyping. Nature reviews. Genetics 12:363-376. DOI: $\underline{10.1038 / \mathrm{nrg} 2958}$. 
239 D, Ménager H, Nedeljkovich M, Scales M, Soiland-Reyes S, Stojanovic L. 2016. Common

240 Workflow Language, v1.0. DOI: 10.6084/m9.figshare.3115156.v2.

241

242 Bal H, Epema D, de Laat C, van Nieuwpoort R, Romein J, Seinstra F, Snoek C, Wijshoff H.

243 2016. A Medium-Scale Distributed System for Computer Science Research: Infrastructure for

244 the Long Term. Computer 49:54-63. DOI: 10.1109/MC.2016.127.

245

246 Becker T, Lee WP, Leone J, Zhu Q, Zhang C, Liu S, Sargent J, Shanker K, Mil-Homens A, 247 Cerveira E, Ryan M, Cha J, Navarro FCP, Galeev T, Gerstein M, Mills RE, Shin DG, Lee C, 248 Malhotra A. 2018. Genome biology 19:38. DOI: 10.1186/s13059-018-1404-6.

249

250 Cameron DL, Schröder J, Penington JS, Do H, Molania R, Dobrovic A, Speed TP, Papenfuss

251 AT. 2017. GRIDSS: sensitive and specific genomic rearrangement detection using positional de

252 Bruijn graph assembly. Genome Research 27:2050-2060. DOI: 10.1101/gr.222109.117.

253

254

Cameron D, Dong R. 2019. StructuralVariantAnnotation: Variant annotations for structural

255

variants. R package (version 1.0.0). https://bioconductor.org.

256

257 Chen X, Schulz-Trieglaff O, Shaw R, Barnes B, Schlesinger F, Källberg M, Cox AJ, Kruglyak S, 258 Saunders CT. 2016. Manta: rapid detection of structural variants and indels for germline and 259 cancer sequencing applications. Bioinformatics 32:1220-1222. DOI:

260 10.1093/bioinformatics/btv710.

261

262 Conway JR, Lex A, Gehlenborg N. 2017. UpSetR: an R package for the visualization of 263 intersecting sets and their properties. Bioinformatics 33:2938-2940. DOI:

264 10.1093/bioinformatics/btx364.

265

266 English AC, Salerno WJ, Hampton OA, Gonzaga-Jauregui C, Ambreth S, Ritter DI, Beck CR, 267 Davis CF, Dahdouli M, Ma S, Carroll A, Veeraraghavan N, Bruestle J, Drees B, Hastie A, Lam 268 ET, White S, Mishra P, Wang M, Han Y, Zhang F, Stankiewicz P, Wheeler DA, Reid JG, Muzny 269 DM, Rogers J, Sabo A, Worley KC, Lupski JR, Boerwinkle E, Gibbs RA. 2015. Assessing 270 structural variation in a personal genome - towards a human reference diploid genome. BMC 271 Genomics 16:286. DOI: 10.1186/s12864-015-1479-3. 
273 Fang L, Hu J, Wang D, Wang K. 2018. NextSV: a meta-caller for structural variants from low274 coverage long-read sequencing data. BMC Bioinformatics 19:180. DOI: 10.1186/s12859-018$275 \underline{2207-1}$.

276

277 Gröbner SN, Worst BC, Weischenfeldt J, Buchhalter I, Kleinheinz K, Rudneva VA, Johann PD, 278 Balasubramanian GP, Segura-Wang M, Brabetz S, Bender S, Hutter B, Sturm D, Pfaff E, 279 Hübschmann D, Zipprich G, Heinold M, Eils J, Lawerenz C, Erkek S, Lambo S, Waszak S, 280 Blattmann C, Borkhardt A, Kuhlen M, Eggert A, Fulda S, Gessler M, Wegert J, Kappler R, 281 Baumhoer D, Burdach S, Kirschner-Schwabe R, Kontny U, Kulozik AE, Lohmann D, Hettmer 282 S, Eckert C, Bielack S, Nathrath M, Niemeyer C, Richter GH, Schulte J, Siebert R, Westermann 283 F, Molenaar JJ, Vassal G, Witt H, ICGC PedBrain-Seq Project, ICGC MMML-Seq Project, Burkhardt B, Kratz CP, Witt O, van Tilburg CM, Kramm CM, Fleischhack G, Dirksen U, J, Resnick AC, Zhang J, Liu Y, Zhou X, Waanders AJ, Zwijnenburg DA, Raman P, Brors B,

287

288

289

290

291

292 293

294 295 296 297 298

299 300 301 302 303 304

Weber UD, Northcott PA, Pajtler KW, Kool M, Piro RM, Korbel JO, Schlesner M, Eils R, Jones DTW, Lichter P, Chavez L, Zapatka M, Pfister SM. 2018. The landscape of genomic alterations across childhood cancers. Nature 555:321-327. DOI: 10.1038/nature25480.

Holmes IH, Mungall CJ. 2017. BioMake: a GNU make-compatible utility for declarative workflow management. Bioinformatics 33:3502-3504. DOI: 10.1093/bioinformatics/btx306.

Jeffares DC, Jolly C, Hoti M, Speed D, Shaw L, Rallis C, Balloux F, Dessimoz C, Bähler J, Sedlazeck FJ. 2017. Transient structural variations have strong effects on quantitative traits and reproductive isolation in fission yeast. Nature Communications 8:14061. DOI: 10.1038/ncomms 14061.

Jiménez RC, Kuzak M, Alhamdoosh M, Barker M, Batut B, Borg M, Capella-Gutierrez S, Chue Hong N, Cook M, Corpas M, Flannery M, Garcia L, Gelpí JL, Gladman S, Goble C, González Ferreiro M, Gonzalez-Beltran A, Griffin PC, Grüning B, Hagberg J, Holub P, Hooft R, Ison J, Katz DS, Leskošek B, López Gómez F, Oliveira LJ, Mellor D, Mosbergen R, Mulder N, PerezRiverol Y, Pergl R, Pichler H, Pope B, Sanz F, Schneider MV, Stodden V, Suchecki R, Svobodová Vařeková R, Talvik H-A, Todorov I, Treloar A, Tyagi S, van Gompel M, Vaughan

Peer] reviewing PDF | (2019:04:37207:2:0:NEW 3 Nov 2019) 
305 D, Via A, Wang X, Watson-Haigh NS, Crouch S. 2017. Four simple recommendations to 306 encourage best practices in research software. F1000Research 6:876. DOI:

$307 \quad 10.12688 /$ f1000research.11407.1.

308

309 Köster J, Rahmann S. 2012. Snakemake--a scalable bioinformatics workflow engine.

310 Bioinformatics 28:2520-2522. DOI: 10.1093/bioinformatics/bts480.

311

312 Kurtzer GM, Sochat V, Bauer MW. 2017. Singularity: Scientific containers for mobility of 313 compute. PloS One 12:e0177459. DOI: 10.1371/journal.pone.0177459.

314

315 Kuzniar A, Maassen J, Verhoeven S, Santuari L, Shneider C, Kloosterman W, de Ridder J. 2018. 316 A portable and scalable workflow for detecting structural variants in whole-genome sequencing 317 data. In: 2018 IEEE 14th International Conference on e-Science (e-Science). Amsterdam: IEEE, 318 303-304. DOI: 10.1109/eScience.2018.00064.

319

320

Kuzniar A. 2019. sv-callers (version 1.1.0) [Software]. Zenodo. DOI: 10.5281/zenodo.1217111.

321

322 Kuzniar A, Santuari L. 2019. Test data for $s v$-callers workflow (version 1.1.0) [Dataset].

323 Zenodo. DOI: $10.5281 /$ zenodo.2663307.

324

325

Layer RM, Chiang C, Quinlan AR, Hall IM. 2014. LUMPY: a probabilistic framework for 326 structural variant discovery. Genome biology 15:R84. DOI: 10.1186/gb-2014-15-6-r84.

327

328

Leipzig J. 2017. A review of bioinformatic pipeline frameworks. Briefings in Bioinformatics 329 18:530-536. DOI: $10.1093 / \mathrm{bib} / \mathrm{bbw} 020$.

330

331

Li H. 2013. Aligning sequence reads, clone sequences and assembly contigs with BWA-MEM. 332 arXiv: 1303.3997.

333

334 Li H, Handsaker B, Wysoker A, Fennell T, Ruan J, Homer N, Marth G, Abecasis G, Durbin R, 3351000 Genome Project Data Processing Subgroup. 2009. The Sequence Alignment/Map format 336 and SAMtools. Bioinformatics 25:2078-2079. DOI: 10.1093/bioinformatics/btp352. 
338 Lin K, Smit S, Bonnema G, Sanchez-Perez G, de Ridder D. 2015. Making the difference:

339 integrating structural variation detection tools. Briefings in Bioinformatics 16:852-864. DOI:

$340 \quad 10.1093 / \mathrm{bib} / \mathrm{bbu} 047$.

341

342 Ma X, Liu Y, Liu Y, Alexandrov LB, Edmonson MN, Gawad C, Zhou X, Li Y, Rusch MC,

343 Easton J, Huether R, Gonzalez-Pena V, Wilkinson MR, Hermida LC, Davis S, Sioson E, Pounds

344 S, Cao X, Ries RE, Wang Z, Chen X, Dong L, Diskin SJ, Smith MA, Guidry Auvil JM, Meltzer

345 PS, Lau CC, Perlman EJ, Maris JM, Meshinchi S, Hunger SP, Gerhard DS, Zhang J. 2018. Pan-

346 cancer genome and transcriptome analyses of 1,699 paediatric leukaemias and solid tumours.

347 Nature 555:371-376. DOI: 10.1038/nature25795.

348

349

Maassen J, Drost N, Hage WV, van Nieuwpoort RV. 2017. Track 2 Lightning Talk: Software

350 Development Best Practices at the Netherlands eScience Center. In: Workshop on Sustainable

351 Software for Science: Practice and Experiences (WSSSPE5.1). DOI:

$352 \quad 10.6084 / \mathrm{m} 9$. figshare.5327587.v2.

353

354

Maassen J, Verhoeven S, Borgdorff J, Spaaks JH, Drost N, Meijer C, van der Ploeg A, de Boer

355

PT, van Nieuwpoort R, van Werkhoven B, Kuzniar A. 2018. Xenon [Software]. Zenodo. DOI:

356

$\underline{10.5281 / \text { zenodo.597993. }}$.

357

358

Merzky A, Weidner O, Jha S. 2015. SAGA: A standardized access layer to heterogeneous

359

Distributed Computing Infrastructure. SoftwareX 1-2:3-8. DOI: 10.1016/j.softx.2015.03.001.

360

361

Mohiyuddin M, Mu, JC, Li J, Bani Asadi N, Gerstein MB, Abyzov A, Wong WH, Lam HYK.

362

2015. MetaSV: an accurate and integrative structural-variant caller for next generation

363

sequencing. Bioinformatics 31:2741-2744. DOI: 10.1093/bioinformatics/btv204.

364

365

Parikh H, Mohiyuddin M, Lam HY, Iyer H, Chen D, Pratt M, Bartha G, Spies N, Losert W,

Zook JM, Salit M. 2016. svclassify: a method to establish benchmark structural variant calls.

BMC Genomics 17:64. DOI: 10.1186/s12864-016-2366-2.

van Nieuwpoort RV, Kielmann T, Bal HE. 2007. User-friendly and reliable grid computing based on imperfect middleware. In: Proceedings of the 2007 ACM/IEEE conference on 
372

373 Rausch T, Zichner T, Schlattl A, Stütz AM, Benes V, Korbel JO. 2012. DELLY: structural

374 variant discovery by integrated paired-end and split-read analysis. Bioinformatics 28:i333-i339.

375 DOI: $10.1093 /$ bioinformatics/bts378.

376

377 Stratton MR. 2011. Exploring the genomes of cancer cells: progress and promise. Science (New 378 York, N.Y.) 331:1553-1558. DOI: 10.1126/science.1204040.

379

380 Troger P, Rajic H, Haas A, Domagalski P. 2007. Standardization of an API for Distributed

381 Resource Management Systems. In: Seventh IEEE International Symposium on Cluster

382 Computing and the Grid (CCGrid '07). Rio De Janeiro: IEEE, 619-626. DOI:

383 10.1109/CCGRID.2007.109.

384

385 da Veiga Leprevost F, Grüning BA, Alves Aflitos S, Röst HL, Uszkoreit J, Barsnes H, Vaudel 386 M, Moreno P, Gatto L, Weber J, Bai M, Jimenez RC, Sachsenberg T, Pfeuffer J, Vera Alvarez R, 387 Griss J, Nesvizhskii AI, Perez-Riverol Y. 2017. BioContainers: an open-source and community388 driven framework for software standardization. Bioinformatics 33:2580-2582. DOI:

389 10.1093/bioinformatics/btx192.

390

391 Verhoeven S, Spaaks JH. 2019. Xenon command line interface [Software]. Zenodo. DOI:

$392 \underline{10.5281 / \text { zenodo.597603. }}$

393

394 Vivian J, Rao AA, Nothaft FA, Ketchum C, Armstrong J, Novak A, Pfeil J, Narkizian J, Deran 395 AD, Musselman-Brown A, Schmidt H, Amstutz P, Craft B, Goldman M, Rosenbloom K, Cline 396 M, O’Connor B, Hanna M, Birger C, Kent WJ, Patterson DA, Joseph AD, Zhu J, Zaranek S, 397 Getz G, Haussler D, Paten B. 2017. Toil enables reproducible, open source, big biomedical data 398 analyses. Nature Biotechnology 35:314-316. DOI: 10.1038/nbt.3772.

399

400 Yung CK, O’Connor BD, Yakneen S, Zhang J, Ellrott K, Kleinheinz K, Miyoshi N, Raine KM, 401 Royo R, Saksena GB, Schlesner M, Shorser SI, Vazquez M, Weischenfeldt J, Yuen D, Butler 402 AP, Davis-Dusenbery BN, Eils R, Ferretti V, Grossman RL, Harismendy O, Kim Y, Nakagawa 403 H, Newhouse SJ, Torrents D, Stein LD. 2017. Large-Scale Uniform Analysis of Cancer Whole 404 Genomes in Multiple Computing Environments. bioRxiv. DOI: 10.1101/161638. 
406 Zook JM, Catoe D, McDaniel J, Vang L, Spies N, Sidow A, Weng Z, Liu Y, Mason CE, 407 Alexander N, Henaff E, McIntyre ABR, Chandramohan D, Chen F, Jaeger E, Moshrefi A, Pham 408 K, Stedman W, Liang T, Saghbini M, Dzakula Z, Hastie A, Cao H, Deikus G, Schadt E, Sebra R, 409 Bashir A, Truty RM, Chang CC, Gulbahce N, Zhao K, Ghosh S, Hyland F, Fu Y, Chaisson M, 410 Xiao C, Trow J, Sherry ST, Zaranek AW, Ball M, Bobe J, Estep P, Church GM, Marks P, 411 Kyriazopoulou-Panagiotopoulou S, Zheng GXY, Schnall-Levin M, Ordonez HS, Mudivarti PA, 412 Giorda K, Sheng Y, Rypdal KB, Salit M. 2016. Extensive sequencing of seven human genomes 413 to characterize benchmark reference materials. Scientific Data 3:160025. DOI:

$414 \quad \underline{10.1038 / \text { sdata.2016.25. }}$. 
Figure 1

Workflow/Xenon software architecture.

(A) The workflow includes four SV callers namely Manta, DELLY, LUMPY and GRIDSS to detect

SVs in (paired) samples given a reference genome. Submitted jobs execute in parallel across compute nodes. SVs detected per caller are filtered and merged into one (union) call set.

Note: DELLY requires separate runs for each SV type (i.e., DUP=duplication, DEL=deletion, INS=insertion, INV=inversion and BND=translocation). (B) The workflow uses the Xenon command-line interface (xenon-cli) that abstracts away scheduler- and file transfer-specific commands; the Xenon library translates each command to the one used by the target system.
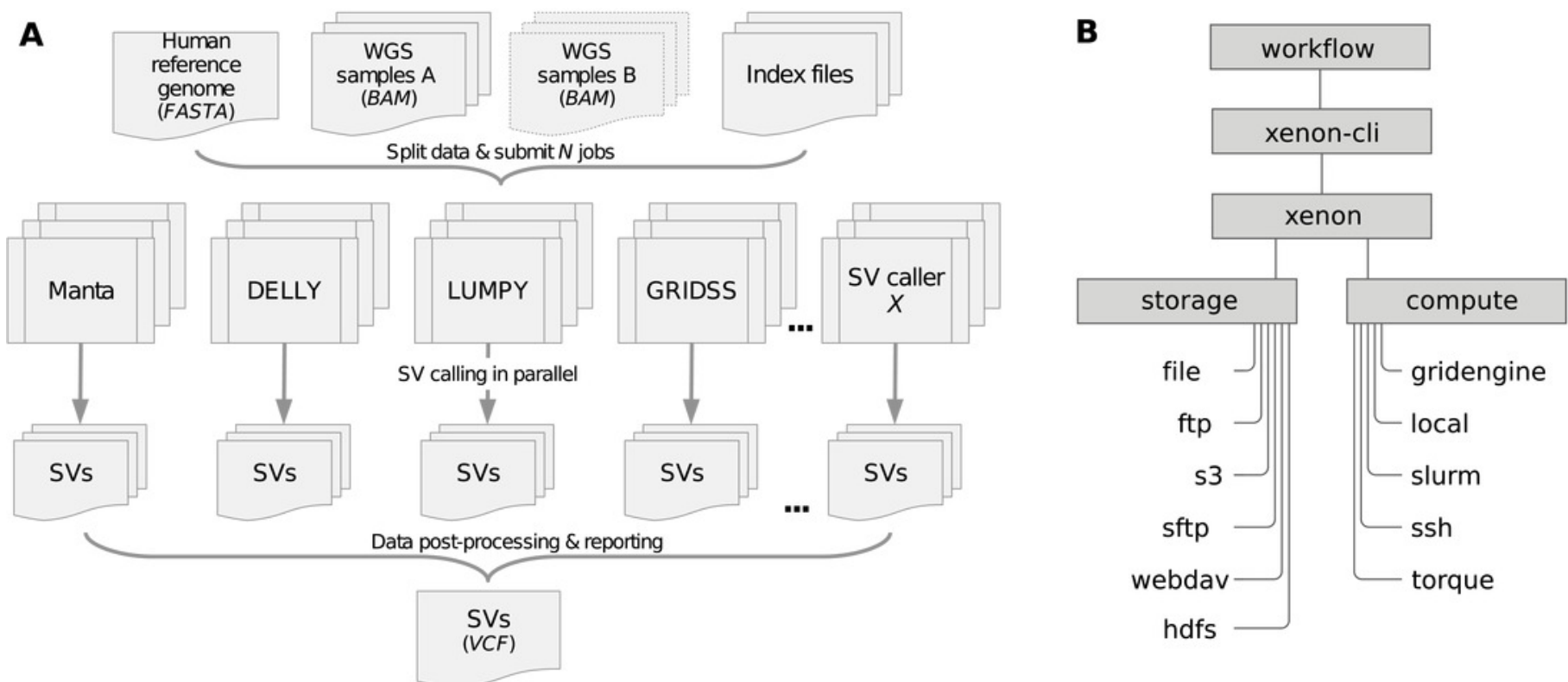


\section{Figure 2}

Figure 2. Venn diagrams of intersecting SV call sets.

(A) Germline and (B) somatic SVs detected in the benchmark and in the cell lines samples, respectively, using Manta, DELLY, LUMPY and GRIDSS. Most SVs are caller-specific, followed by SVs common to three of the four callers. SVs detected by the callers were filtered and merged into one set (see the Methods section). Note: The Venn diagrams include the largest GRIDSS sets as the GRIDSS output varies slightly each run using the same input. Fig. S1-2 show the comparisons across sample copies.

A

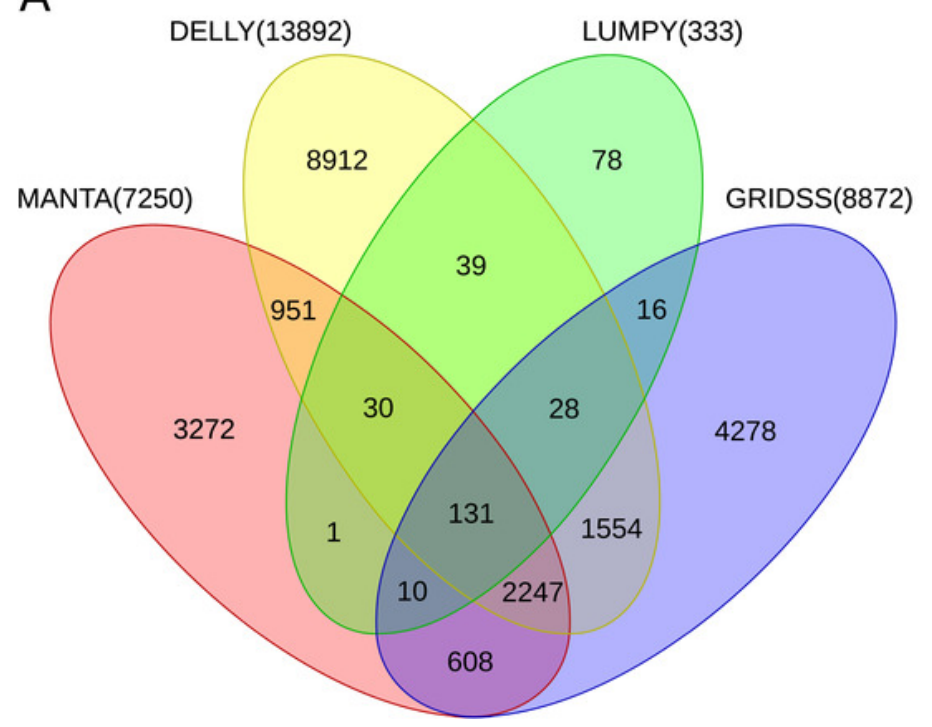

B

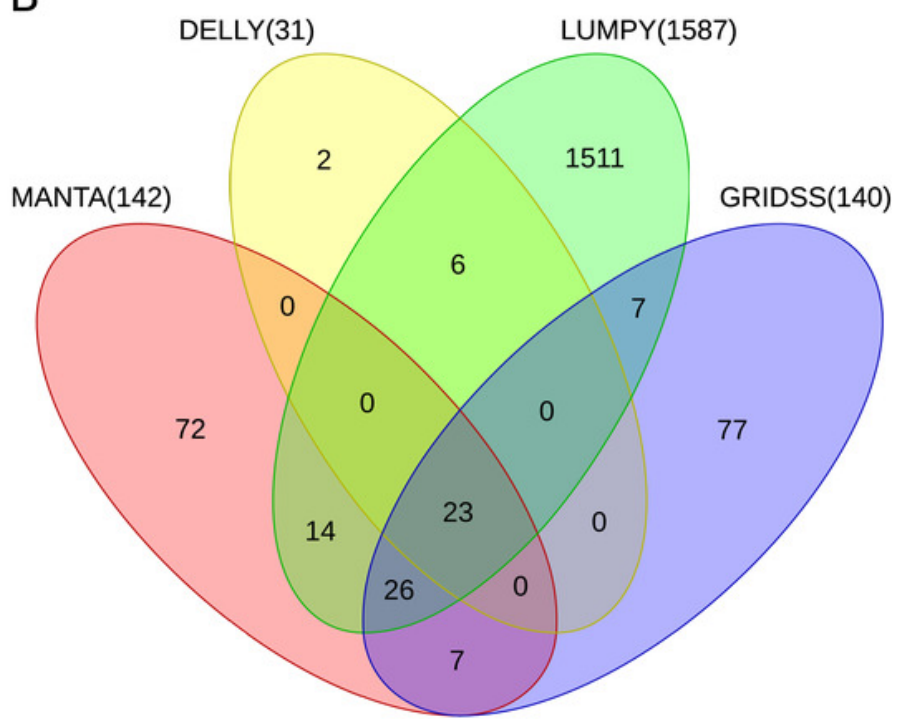


Figure 3

Precision and recall of SV detection based on a single versus multiple caller(s) according to two truth sets.

(A) For Personalis/1kGP data, the merged calls of at least three SV callers (denoted as 'merge3+') have best precision (88.1\%) while DELLY calls have best recall (85.3\%). (B) For PacBio/Moleculo data, the 'merge3+' calls have best precision (94.8\%) while Manta calls have best recall (63\%).

A

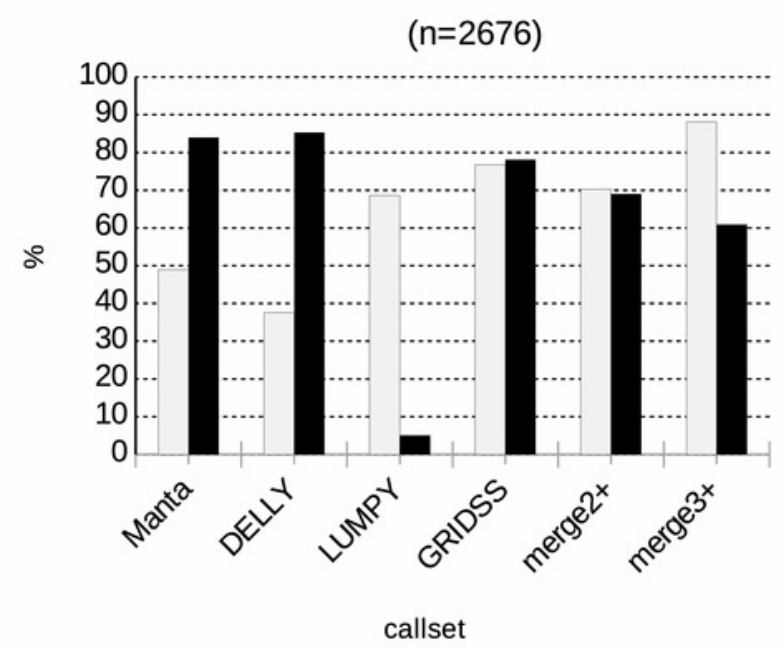

B

PacBio/Moleculo

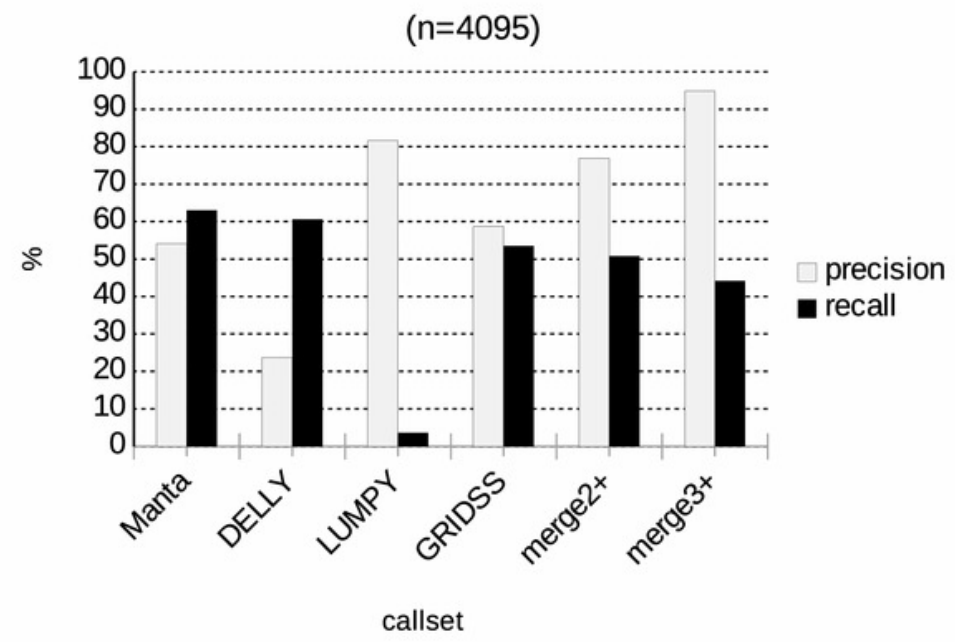




\section{Table 1 (on next page)}

SV detection tools included in the workflow. 
1 Table 1. SV detection tools included in the workflow.

\begin{tabular}{l|l|l|l|}
\hline Software & Implementation & Parallelism & I/O files \\
\hline Manta & C ++ , Python & pyFlow tasks ${ }^{1}$, SIMD $^{2}$ & FASTA, BAM or CRAM / VCF \\
\hline DELLY & C ++ & OpenMP threads & - \\
\hline LUMPY & C/C,++ Python & FASTA, BAM / BCF \\
\hline GRIDSS & Java, R, Python & FASTA, BAM / VCF \\
\hline $\begin{array}{l}\text { 'http://illumina.github.io/pyflow; }{ }^{3} \text { Single Instruction Multiple Data vector processing; }{ }^{3} \text { depends on the number of input samples used (i.e., max. } 2 \text { in the paired-sample } \\
\text { mode). }\end{array}$
\end{tabular}

2 'http://illumina.github.io/pyflow; ${ }^{2}$ Single Instruction Multiple Data vector processing; ${ }^{3}$ depends on the number of input samples used (i.e., max. 2 in the paired-sample
mode). 
Table 2 (on next page)

Compute resources used by the SV callers. 
1 Table 2. Compute resources used by the SV callers.

\begin{tabular}{|l|l|l|l|l|l|}
\hline Software & Dataset & $\begin{array}{l}\text { Threads } \\
\text { per job }\end{array}$ & $\begin{array}{l}\text { Wall-clock/CPU } \\
\text { times* }(\text { HH:MM) }\end{array}$ & Peak mem. (GiB) & Output \\
\hline Manta & cell lines & 24 & $01: 45 / 14: 31$ & 5.3 & $94 \mathrm{MiB}$ \\
\hline & benchmark & 24 & $00: 49 / 05: 42$ & 2.8 & $225 \mathrm{MiB}$ \\
\hline DELLY & cell lines & 2 & $\frac{19: 23 / 25: 47}{66: 03 / 85: 20}$ & 3.6 & $13 \mathrm{MiB}$ \\
& benchmark & 1 & $\underline{00: 54 / 00: 49}$ & 0.9 & $28 \mathrm{MiB}$ \\
\hline LUMPY & cell lines & 1 & $13: 07 / 08: 35$ & 24.4 & $5.9 \mathrm{GiB}$ \\
\hline & benchmark & 1 & $00: 29 / 00: 45$ & 5.7 & $<1 \mathrm{MiB}$ \\
\hline GRIDSS & cell lines & 24 & $29: 31 / 214: 13$ & 44.4 & $73 \mathrm{GiB}$ \\
\hline & benchmark & 24 & $08: 24 / 26: 04$ & 38.5 & $342 \mathrm{MiB}$ \\
\hline
\end{tabular}

